\title{
EFEITOS DA INGESTÃO DE SOLUÇÃO CARBOIDRATADA E ÁGUA SOBRE A MASSA CORPORAL, HEMATÓCRITO E VOLUME DE URINA EM PRATICANTES DE CICLISMO INDOOR
}

\author{
Ingestion effects of electrolytic carbohydrated solution and water, on corporal mass, \\ hematocrit and urinary volume in indoor cycling practicing
}

\author{
Juliano Gross da Costa ${ }^{1}$, Bianca da Silva ${ }^{1}$, César Augusto Otero Vaghetti ${ }^{2}$
}

\section{Resumo}

O ciclismo indoor é realizado em bicicletas específicas, onde as capacidades físicas, resistência e velocidade, são evidenciadas. $O$ objetivo da pesquisa foi identificar alterações na massa corporal, no hematócrito e no volume urinário, em sete indivíduos do gênero masculino, praticantes de ciclismo indoor, antes (pré-teste) e depois (pós-teste) de serem submetidos a três situações distintas, cada qual separada por um intervalo de $48 \mathrm{~h}$ : ingestão de $400 \mathrm{ml}$ de solução carboidratada eletrolítica a $6 \%$ (SE), 400ml de água (AG) e sem ingestão de líquidos ( $\mathrm{SH}$ ), durante uma aula de ciclismo indoor (50min), verificando as diferenças estatísticas entre esses grupos. Os testes foram aplicados em um ambiente com temperatura média de $25^{\circ} \mathrm{C}$ e umidade relativa do ar média de $85 \%$. O exercício físico foi realizado em um cicloergômetro, no qual o padrão de movimento constou de 50 minutos de exercício, a $85 \%$ da freqüência cardíaca máxima, com intervalos de um minuto de recuperação ativa a cada 20 minutos, com intensidade e duração do exercício idêntico às utilizadas em aulas de ciclismo indoor. Os resultados indicaram perdas de massa corporal, alterações no hematócrito, assim como variações no volume urinário, respectivamente, de 1,47kg(SE), 1,50kg(AG) e 1,31 kg(SH), $1,97 \%$ (SE), $1,48 \%$ (AG) e $2,27 \%$ (SH) e $48,57 \mathrm{ml}(\mathrm{SE})$, $40 \mathrm{ml}(\mathrm{AG})$ e $40,71 \mathrm{ml}(\mathrm{SH})$ para as três situações estudadas. Foram encontradas diferenças estatisticamente significativas, entre o pré-teste e o pós-teste, para cada situação investigada e, entre as situações investigadas, (SE), (AG) e (SH), não foram encontradas diferenças estatisticamente significativas com $p \leq 0,05$.

Palavras-chave: Massa Corporal, Hematócrito, Volume Urinário, Ciclismo Indoor.

\begin{abstract}
The indoor cycling is carried out in specific bicycles, where the physical capacities resistance and speed are evidenced. The objective of the research was to identify alterations in the corporal mass, hematocrit and urinary volume in a group with seven male in indoor cycling practicing before (pre-test) and after (pos-test). They are submitted to three distinct situations which were separated by a break of $48 \mathrm{~h}$ : ingestion of $400 \mathrm{ml}$ of $6 \%$ electrolytic carbohydrated solution (SE), $400 \mathrm{ml}$ of water (AG) and no liquid ingestion (SH) in an indoor cycling (50mim) and verify the statistical differences between those groups. The tests were applied in a $25^{\circ} \mathrm{C}$ average temperature environment and $85 \%$ average of air relative humidity. The physical exercise was carried out in a cycloergometer in which the standard movement consisted of 50 minutes of exercise at $85 \%$ of the maximum cardiac frequency, with intervals of one (1) minute of active recovery at each 20 minutes, with exercise intensity and duration identical to them utilized in classes of indoor cycling. The results indicated losses of corporal mass, hematocrit alterations and urinary volume variations, respectively, of $1.47 \mathrm{~kg}(\mathrm{SE}), 1.50 \mathrm{~kg}(\mathrm{AG})$ and $1.31 \mathrm{~kg}(\mathrm{SH}), 1.97 \%(\mathrm{SE}), 1.48 \%(\mathrm{AG})$ and $2.27 \%(\mathrm{SH})$ and $48.57 \mathrm{ml}(\mathrm{SE}), 40 \mathrm{ml}(\mathrm{AG})$ and $40.71 \mathrm{ml}(\mathrm{SH})$ for the three situations studied. Significant statistical differences were found between the pre-test and the pos-test for each situation investigated and, between the situations investigated (SE), $(\mathrm{AG})$ and $(\mathrm{SH})$, there were no significant statistical differences with $p \leq 0.05$.
\end{abstract}

Key words: Corporal Mass, Hematocrit, Urinary Volume, Indoor Cycling

1. Universidade Gama Filho - Rio de Janeiro - RJ - Brasil.

2. Universidade Federal de Pelotas - Pelotas - RS - Brasil.

Revista de Educação Física 2008 Dez; 143:12-17. Rio de Janeiro (RJ) - Brasil.

Recebido em 07.06.2008. Aceito em 15.09.2008. 


\section{INTRODUÇÃO}

A água representa, aproximadamente, $60 \%$ da massa corporal de um indivíduo adulto, podendo esse valor variar de acordo com o sexo, a idade, o condicionamento físico, a composição corporal e os estoques de glicogênio muscular ${ }^{1}$. Essa molécula é um importante nutriente, está presente em ambientes intra-celulares e extra-celulares e, segundo Sawka ${ }^{2}$, sua ausência compromete profundamente a função cardiovascular e a capacidade de realizar exercício físico.

A desidratação é definida, por Robergs e Roberts ${ }^{3}$, como a diminuição do conteúdo de água corporal abaixo dos níveis de normalidade, decorrente de um processo fisiológico responsável pela termorregulação corporal, onde o organismo faz trocas de calor com o meio ambiente, perdendo água pela respiração e pela transpiração. Juntamente com a eliminação de líquido corporal, alguns eletrólitos, como sódio e potássio, responsáveis pela manutenção da osmolalidade dos meios intra e extracelulares, também são eliminados ${ }^{2}$. Até mesmo níveis baixos de desidratação, variando de um a dois por cento da massa corporal, podem causar distúrbios fisiológicos, como a redução do volume plasmático, alterações eletrolíticas e deficiência no controle da temperatura corporal, reduzindo o desempenho durante o exercício físico ${ }^{4}$.

Os músculos são verdadeiras usinas produtoras de calor ${ }^{5} \mathrm{e}$, portanto, a evaporação constitui a principal defesa fisiológica contra o superaquecimento. Em resposta ao estresse térmico, induzido por determinada atividade física ou exercício físico, as glândulas écrinas secretam quantidades de solução hipotônica, as quais têm a função de resfriar a pele, transferindo o calor para o meio por evaporação. Dessa forma, a pele resfria o sangue periférico, permitindo que possa retornar aos tecidos mais profundos, a fim de captar mais calor e, à medida que a desidratação progride, o volume plasmático diminui, o fluxo sangüíneo periférico e o ritmo de transpiração são reduzidos, tornando a termorregulação mais difícil ${ }^{6}$.

Alguns fatores podem acelerar os processos de desidratação, como a temperatura ambiente, a umidade relativa do ar, a intensidade do exercício físico realizado, o nível de condicionamento físico e o estado de hidratação inicial. Para tentar evitar uma perda de líquido muito acentuada, Naves et al. ${ }^{7}$ afirmam que o organismo ativa, como meio de proteção, um complexo sistema de controle do balanço hidroeletrolítico e da constância da osmolalidade, que envolve a produção do hormônio antidiurético, sistema renina angiotensina aldosterona e o mecanismo da sede. O objetivo primário da reposição líquida consiste em manter o volume plasmático, para que a circulação e a transpiração possam prosseguir em níveis ideais, possibilitando um bom funcionamento do sistema de termorregulação ${ }^{6}$. Portanto, para atenuar os efeitos da desidratação, é essencial a ingestão de líquidos, de água ou de repositores eletrolíticos, durante a prática de exercícios físicos.

O ciclismo indoor $(\mathrm{Cl})$ é uma prática muito comum nas academias de ginástica. Realizado em bicicletas específicas ou ergométricas, essa modalidade caracteriza-se como um exercício aeróbio de alta intensidade, que promove o desenvolvimento do sistema cardiorrespiratório e um elevado gasto calórico, onde as capacidades físicas, resistência e velocidade de movimento dos membros inferiores, são evidenciadas. Durante a prática dessa modalidade, é comum observar uma grande produção de suor nos praticantes. Alguns ingerem água e bebidas eletrolíticas vendidas comercialmente, e outros, não costumam consumir nenhum tipo de líquido durante a prática do exercício. Dessa forma, objetivou-se verificar os efeitos da ingestão de solução carboidratada eletrolítica a $6 \%(\mathrm{SE})$ e água (AG), sobre a variação da massa corporal (MC), hematócrito (HCT) e volume de urina (VU), em praticantes de ciclismo indoor.

\section{MÉTODO}

Sete indivíduos, do gênero masculino, participaram da pesquisa, praticantes de $\mathrm{Cl}$ em academia e treinados na modalidade com mais de seis meses de prática. Os sujeitos foram orientados sobre os procedimentos da pesquisa e, também, assinaram Termo de Consentimento para a realização do experimento.

Fez-se necessário, para o desenvolvimento do experimento, água mineral (marca Ouro Verde), solução carboidratada eletrolítica a $6 \%$ e $90 \mathrm{mg}$ de sódio (marca Gatorade), proveta de plástico de 100ml (marca J. Prolab) e copo becker de plástico (marca J. Prolab) de 250 (ml), frequencímetros cardíacos (marca Polar-A1), termômetro de mercúrio (marca Incoterm), termo-higrômetro (marca Hygrotherm), desumidificador de ar (marca Artel, modelo B-EA16MD19), ar condicionado (marca Springer), balança (marca Filizola, modelo 31) com sensibilidade de $0,1 \mathrm{~kg}$, 
bicicletas de ciclismo Indoor (marca World W6), agulhas e seringas descartáveis de sangue venoso $3 \mathrm{ml}$, anticoagulante EDTA 10\% (ácido etilendiaminotetracético dissódico), equipamento de automação multiparamétrica em hematologia (marca Micros - ABX 60) e um plicômetro (marca Cescorf, modelo 4107) para mensurar o percentual de gordura, segundo Guedes ${ }^{8}$.

A coleta dos dados foi realizada na academia Spazio (Pelotas-RS), em um ambiente com temperatura média de $25^{\circ} \mathrm{C}$ e umidade relativa do ar média de $85 \%$. Os procedimentos de coleta foram divididos em três etapas, cada qual separada por um intervalo de 48 horas, nas quais, antes (pré-teste) e depois (pós-teste) do exercício físico, foram coletados urina e sangue, sendo, também, mensurada a massa corporal. Os exames de HCT foram analisados no laboratório Gorgot, na mesma cidade. A intensidade e duração do exercício realizado foram idênticas às utilizadas em aulas de $\mathrm{Cl}$, nas quais o padrão de movimento na bicicleta constou de 50 minutos de exercício a $85 \%$ da freqüência cardíaca máxima individual, mensurada segundo os protocolos de Tanaka, Monahan e Seals ${ }^{9}$, e intervalos de um minuto de recuperação ativa a cada 20 minutos. Na primeira e segunda etapa da coleta de dados, os sujeitos receberam, respectivamente, SE e AG, ambos a um volume de $400 \mathrm{ml}$, fracionados em duas porções de $200 \mathrm{ml}$, a cada intervalo de recuperação ativa durante a prática do $\mathrm{Cl}$. Na terceira etapa, repetiu-se o procedimento das duas etapas anteriores, porém a cada intervalo de recuperação não foi feito nenhum tipo de hidratação (SH).
Foi utilizada uma estatística descritiva com média e desvio padrão (DP) para descrever as características da amostra, da idade, da massa corporal, da estatura, da gordura corporal e da freqüência cardíaca. Dois testes de hipóteses também foram utilizados: o teste " $t$ " de student pareado, para verificar diferenças entre as médias do pré-teste e o pós-teste em cada situação investigada; e o teste " $t$ " de student independente, para verificar as diferenças entre as médias das variações nas três situações investigadas SE, AG e SH. Os cálculos foram processados no programa Excel 2003, no qual foi utilizado um valor de significância $p \leq 0,05$.

\section{RESULTADOS}

Na TABELA 1, pode-se observar as características da amostra $(n=7)$, sendo apresentados os resultados médios e desvio padrão.

Na TABELA 2, podem ser observados: média, desvio padrão e valores de probabilidades das variáveis MC, HCT e VU, no pré-teste (pré), no pós-teste (pós), bem como a

TABELA 1

CARACTERÍSTICAS DA AMOSTRA.

\begin{tabular}{lcccccc}
\hline & & & \multicolumn{5}{c}{$\begin{array}{c}\text { Freqüência Freqüência } \\
\text { cardíaca } \\
\text { Idade } \\
\text { (anos) }\end{array}$} & $\begin{array}{c}\text { MC } \\
\text { (kg) }\end{array}$ & $\begin{array}{c}\text { Estatura } \\
\text { (cm) }\end{array}$ & $\begin{array}{c}\text { Corporal } \\
\text { (\%) }\end{array}$ & $\begin{array}{c}\text { repouso } \\
\text { (bpm) }\end{array}$ & $\begin{array}{c}\text { máxima } \\
\text { (bpm) }\end{array}$ \\
\hline Média & 23,14 & 83,08 & 178 & 15,82 & 62 & 196 \\
DP & 2,54 & 10,50 & 6,52 & 2,50 & 6,89 & 2,54 \\
\hline
\end{tabular}

TABELA 2

MÉDIA, DESVIO PADRÃO E PROBABILIDADE DAS VARIÁVEIS: MASSA CORPORAL, HEMATÓCRITO E VOLUME URINÁRIO, NAS TRÊS SITUAÇÕES INVESTIGADAS.

\begin{tabular}{|c|c|c|c|c|c|c|c|c|c|c|}
\hline & & \multicolumn{9}{|c|}{ Situações investigadas } \\
\hline & & \multicolumn{3}{|c|}{ Solução carboidratada (SE) } & \multicolumn{3}{|c|}{ Água (AG) } & \multicolumn{3}{|c|}{ Sem hidratação (SH) } \\
\hline & & Pré & Pós & Var 1 & Pré & Pós & Var 2 & Pré & Pós & Var 3 \\
\hline \multirow[t]{3}{*}{$\mathrm{MC}(\mathrm{kg})$} & Média & 83,48 & 82,01 & 1,47 & 83,40 & 81,90 & 1,50 & 82,93 & 81,61 & 1,31 \\
\hline & $\mathrm{DP} \pm$ & 10,50 & 10,01 & & 10,52 & 10,06 & & 10,33 & 10,02 & \\
\hline & $\mathrm{p}$ & \multicolumn{2}{|c|}{$0,00124^{\star}$} & & \multicolumn{2}{|c|}{$0,00121^{*}$} & & \multicolumn{2}{|c|}{$0,00008^{*}$} & \\
\hline \multirow[t]{3}{*}{ HCT (\%) } & Média & 41,61 & 43,59 & 1,98 & 41,69 & 43,17 & 1,48 & 40,87 & 43,14 & 2,27 \\
\hline & $\mathrm{DP}_{ \pm}$ & 2,68 & 2,39 & & 3,11 & 1,77 & & 2,74 & 2,60 & \\
\hline & $\mathrm{p}$ & & & & & 40 & & 0,0 & $7^{*}$ & \\
\hline \multirow[t]{2}{*}{ VU (ml) } & Média & & 48,57 & & & 40,00 & & & 43,57 & \\
\hline & $\mathrm{DP}_{ \pm}$ & & 30,65 & & & 21,21 & & & 42,40 & \\
\hline
\end{tabular}


variação ocorrida (Var), respectivamente, Var-1, Var-2 e Var-3 para as três situações investigadas: SE, AG e SH.

Foram encontradas diferenças estatisticamente significativas, com $p \leq 0,05$, entre o pré-teste e o pós-teste, nas três situações investigadas para a variáveis $\mathrm{MC}$, assim como diferenças estatisticamente significativas entre o pré-teste e o pós-teste para SE e SH para a variável HCT, como pode-se observar na TABELA 2. Entretanto, não foram encontradas diferenças significativas, quando comparadas as médias das variações ocorridas nas três situações investigadas.

\section{DISCUSSÃO}

Foi utilizado, nessa pesquisa, um volume de $400 \mathrm{ml}$, segundo as recomendações do American College of Sports Medicine ${ }^{10}$, no qual é recomendado o uso de volumes entre 400 a $800 \mathrm{ml}$ de líquido a cada hora, para hidratação durante o exercício. Mitchell et al. ${ }^{11}$ e Gisolfi et al. ${ }^{12}$ afirmam que volumes de 500 a $800 \mathrm{ml}$ de água, ou de solução, são bem absorvidos em uma hora de atividade física. Entretanto, o volume de líquidos adotado (SE e AG), para a realização da pesquisa, não foi suficiente para evitar que os indivíduos atingissem o grau de $1 \%$ na perda de massa corporal.

Em contrapartida, Walsh et al. ${ }^{13}$ obtiveram resultados semelhantes em relação à perda de massa corporal, sem hidratação, quando utilizado 1 litro de solução por hora, fracionados em $400 \mathrm{ml}$, antes do teste, e $600 \mathrm{ml}$, durante o teste, encontrando diferenças significativas. Maughan e Rehrer ${ }^{14}$ afirmam que o volume de ingestão de líquido influencia, diretamente, na sua absorção gástrica. O volume de líquido ingerido nessa pesquisa foi fracionado em $200 \mathrm{ml}$ para cada intervalo de recuperação, o que minimizou, portanto, a possibilidade de ocorrer desconforto estomacal.

Analisando os dados da variável massa corporal, as variações ocorridas entre as situações onde houve hidratação (SE e AG) foram maiores que a variação onde não houve hidratação (SH). Esses resultados podem estar relacionados com uma diminuição na taxa de transpiração, provocada por uma menor resposta da glândula sudorípara, em conjunto com o aumento da osmolalidade sangüínea, que funciona como um mecanismo de feedback corretivo, que deve manter os líquidos no corpo, quando os mesmos não estão sendo repostos. Embora não tenham sido encontradas diferenças estatisticamente significativas, entre as variações, percebe-se que os valores obtidos de $1,47 \mathrm{~kg}, 1,50 \mathrm{~kg}$ e $1,31 \mathrm{~kg}$ representam, respectivamente, $1,76 \%, 1,79 \%$ e $1,58 \%$ de perda da massa corporal em água, a partir do repouso dos sujeitos. Segundo McArdle et al. ${ }^{6}$, níveis leves de desidratação de 1 a $2 \%$ podem elevar a temperatura do corpo em $0,4\left({ }^{\circ} \mathrm{C}\right)$ para cada percentual subseqüente, podendo prejudicar a termorregulação do organismo e diminuir o desempenho aeróbio. Corroborando com estas afirmações, Montain e Coyle ${ }^{15}$ afirmam que as variações de massa corporal podem acarretar em um aumento de 0,1 a $0,25\left({ }^{\circ} \mathrm{C}\right)$ na temperatura corporal para cada déficit de massa perdida.

Devido à diminuição da volemia, Sallis ${ }^{16}$ afirma que a redução do fluxo de sangue nos vasos subcutâneos dificulta a troca de calor com o ambiente. Embora algumas pesquisas tenham demonstrado variações na temperatura corporal, em diferentes níveis de desidratação, para Meyer e Andrade ${ }^{17}$, ainda não existe um consenso sobre os mecanismos que levam a essa elevação da temperatura.

Foram encontrados valores superiores do HCT, no pós-teste, quando comparados aos resultados obtidos no pré-teste, provavelmente devido a uma redução do volume de água contida no plasma e a um aumento da osmolalidade. Nas três situações propostas, pode-se observar essa variação, ou seja, mesmo tendo uma perda em torno de 1,58 a 1,79 (\%) de massa corporal, esses valores são suficientes para causar uma redução de volume plasmático ${ }^{1}$. No caso da etapa onde não houve hidratação (SH), a variação média de HCT foi de $+2,27 \%$, em valores absolutos, o que representa uma variação de $5,6 \%$ em relação aos níveis de repouso, diferente de valores encontrados por Dill e Costill ${ }^{18}$, que obtiveram resultados de $+1,6 \%$ de variação do $\mathrm{HCT}$, em relação aos índices de repouso, durante um teste onde os sujeitos atingiram $4 \%$ de perda de massa corporal.

Nas etapas da pesquisa SE e AG, foram encontrados, respectivamente, aumentos médios no $\mathrm{HCT}$ de $+1,97 \mathrm{e}$ $+1,48 \%$, o que corresponde a 4,56 e $3,6 \%$ de variação em relação aos níveis de repouso. Embora não tenham sido encontradas diferenças estatisticamente significativas, para as médias das variações no HCT, entre as situações investigadas, esperava-se que os valores de HCT, da etapa em que foi utilizada água para hidratação, apresentasse resultados maiores ou, pelo menos, iguais à etapa onde foi utilizada solução eletrolítica carboidratada.

Embora os cálculos estatísticos demonstrem não haver diferenças significativas, a perda de massa corporal foi levemente maior em AG, tendo o HCT sofrido a menor 
variação, supostamente por esse valor estar relacionado com uma maior hemodiluição em relação à etapa SE, devido ao tempo de ajuste da osmolalidade do plasma, dificultando o redirecionamento dos líquidos para o meio intracelular. O organismo, segundo Sawka ${ }^{2}$, quando em estado de desidratação, o volume plasmático torna-se hipertônico, causando uma redistribuição da água ingerida, que é uma solução hipotônica, entre os compartimentos intra e extra celular. González-Alonso et al. ${ }^{19}$ relatam que a diminuição do volume plasmático acaba tornando o sangue mais viscoso, reduzindo o volume de ejeção e causando um aumento na freqüência cardíaca para manter um débito cardíaco exigido em determinada atividade física. Outros pesquisadores, como Heaps et al. ${ }^{20}$, encontraram variações significativas no volume plasmático, com diminuição de $15 \%$ e, na massa corporal, uma perda de 0,9 a 2,8\%, causando variações na freqüência cardíaca de até 18 batimentos por minuto e, no volume de ejeção, de até $18 \mathrm{ml}$ por batimento, comparando as respostas nos sujeitos hidratados.

A produção de urina foi menor na etapa AG, embora os valores não tenham diferenças significativas. Provavelmente, essa diminuição esteja relacionada ao aumento na secreção do Hormônio Antidiurético (ADH), pelo hipotálamo, e, também, pela ação do sistema renina-angiotensina aldosterona, responsável por fazer a captação de sódio, pois, de acordo com Guyton ${ }^{21}$, esse sistema de regulação só começa a ter grandes influências a partir dos 45 minutos, pois envolve uma seqüência de eventos lentos e complexos.

A secreção do ADH pode ser controlada, basicamente, por um sistema osmótico e um outro volêmico. Segundo Naves et al. ${ }^{7}$, o sistema osmótico começa a ser ativado quando os osmorreceptores detectam uma variação de apenas $2 \%$ na osmolalidade, enquanto o sistema volêmico, através dos barorreceptores, é requisitado quando houver quedas de 8 a 10\% na volemia. Os diferentes volumes de urina, encontrados para cada situação investigada, não foram estatisticamente significantes. Dessa forma, pode existir uma relação com a ação do $\mathrm{ADH}$ em manter o volume plasmático, já que o nível de desidratação, nas três situações propostas, foi semelhante.

\section{CONCLUSÃO}

O volume de $400 \mathrm{ml}$ de líquido, tanto a solução carboidratada, quanto a água, utilizado nessa pesquisa para hidratação, não foi suficiente para evitar um nível leve de desidratação. Portanto, em atividades aeróbias de alta intensidade, em academias, como é o caso do ciclismo indoor, recomenda-se o uso de volumes superiores.

Os valores médios das variáveis massa corporal e hematócrito sofreram alterações no pós-teste, indicando um processo leve de desidratação, nas três situações propostas, hidratação com 400ml de solução carboidratada eletrolítica, a $6 \%$ ou com mesma quantidade de água e sem hidratação, durante 50min de cliclismo indoor. $\mathrm{O}$ volume de urina produzido teve uma pequena variação entre o teste realizado com solução carboidratada eletrolítica a $6 \%$, em comparação com os outros dois testes, hidratação com água e sem hidratação. Não foram encontradas diferenças estatisticamente significativa para as médias das variações nas variáveis estudadas, entre as três situações propostas.

\section{REFERÊNCIAS BIBLIOGRÁFICAS}

1. Marquenzi ML, Lancha Jr AH. Estratégias de reposição hídrica: revisões e recomendações aplicadas. Revista Paulista de Educação Física 1998; 12: 219-27.

2. Sawka MN. Physiological consequences of hypohydration: exercise performance and termoregulation. Med Sci Sports Exerc 1992; 24: 657-70.

3. Robergs Ra, Roberts SO. Princípios fundamentais de fisiologia do exercício para aptidão, desempenho e saúde. São Paulo: Phorte; 2002.

4. Walsh RM, Noakes TD, Hawley JA, Dennis SC. Impaired high-intensity cycling performance time at low levels of dehydration. Int J Sports Med 1994; 15: 392-8.

5. McGinnis PM. Biomecânica do esporte e exercício. Porto Alegre: Artmed; 2002.

6. McArdle WD, Katch FI, Katch VL. Fisiologia do exercício, energia, nutrição e desempenho humano. Rio de Janeiro: Guanabara Koogan; 1998. 
7. Naves LA, Vilar L, Costa ACF, Domingues L, Casulari LA. Distúrbios na secreção e ação do hormônio antidiurético. Arq Bras Endocrinol Metab 2003; 47: 467-81.

8. Guedes DP. Composição corporal: princípios, técnicas e aplicações. 2ª ed. Londrina: APEF; 1994

9. Tanaka H, Monahan KD, Seals DR. Age-predicted maximal heart rate revised. Journal of the American College of Cardiology 2001; 37: 153-6.

10. Sawka MN, Burke LM, Eichner ER, Maughan RJ, Montain SJ, Stachenfeld NS. American College of Sports Medicine Position Stand. Exercise and fluid replacement. Med Sci Sports Exerc 2007; 39: 377-90.

11. Mitchell JB, Grandjean PW, Pizza FX, Starling RD, Holtz RW. The effect of volume ingested on rehydration and gastric emptying following exercise-induced dehydration. Med Sci Sports Exerc 1994; 26: 1135-43.

12. Gisolfi CV, Spranger KJ, Summers RW, Schedl HP, Bleiler TL. Effects of cycle exercise on intestinal absorption in humans. J Appl Physiol 1991; 71: 2518-27.

13. Walsh RM, Noakes TD, Hawley JA, Dennis SC. Impaired high-intensity cycling performance time at low levels of dehydration. Int J Sports Med 1994; 15: 392-8.

14. Maughan RJ, Rehrer NJ. Gastric emptying during exercise. Sports Science Exchange 1994; 7: 46-52.

15. Montain SJ, Coyle EF. Fluid ingestion during exercise increases skin blood flow independent of increases in blood volume. J Appl Physiol 1992; 73: 903-10.

16. Sallis R. Collapse in the endurance athlete. Sports Science Exchange. 2004; 17: 95-103.

17. Meyer F, Andrade R. Desidratação e importância da reposição de líquidos durante o exercício prolongado no calor. Revista Perfil 1999; 3: 12-5.

18. Dill DB, Costil DL. Calculation of percentage changes in volume, of blood, plasma, and red cells in dehydration. J Appl Physioly 1974; 37: 247-8.

19. González-Alonso J, Mora-Rodríguez R, Below PR, Coyle EF. Dehydrations markedly impairs cardiovascular function in hyperthermic in endurance athletes during exercise. J Appl Physiol 1997; 82: 1229-36.

20. Heaps CL, González-Alonso J, Coyle EF. Hypohydration causes cardiovascular drift without reducing blood volume. Int J Sports Med 1994; 15: 74-9.

21. Guyton AC. Tratado de Fisiologia Médica. 8ª ed. Rio de Janeiro: Guanabara Koogan; 1992.

\section{Endereço para correspondência:}

César Augusto Otero Vaghetti Rua Fernando Osório, 511 - Bairro Cassino Cidade Rio Grande - RS - Brasil Tel.: (53) 3236-1262 / 91619220 e-mail: cesarvaghetti@ hotmail.com 